\title{
Nonequilibrium Dissolution-diffusion Model for PDMS Membrane Pervaporation of ABE Water Binary System
}

\author{
Xia Yang ${ }^{1}$, Zhen $\mathbf{W u}^{2}$, Fang Manquan ${ }^{1}$ and Li Jiding ${ }^{1 *}$ \\ ${ }^{1}$ State Key Laboratory of Chemical Engineering, Department of Chemical Engineering, Tsinghua University, Beijing 100084, China \\ ${ }^{2}$ Ordos Redbud Innovation Institute, Ordos 017000, China
}

\begin{abstract}
Previous models of equilibrium dissolution-diffusion, pore flow and virtual phase change cannot describe the mass transfer process of pervaporation precisely. The fact that dissolution process on the surface of the membrane does not reach equilibrium is seldom emphasized in the literature. The aim of the present work is to develop the nonequilibrium dissolution-diffusion model (nonequilibrium model) for membrane pervaporation process. In this research, the steps of dissolution and desorption were treated as the pseudo surface reaction processes on the surface based on the hypothesis of nonequilibrium dissolution at the interface of the feed liquid and membrane. The semi-experimental model was set based on steady state mass transfer, ignoring the concentration polarization and adsorption at the permeation side. Through linear fitting of the flux with different thickness of the membrane, the diffusion coefficients and adsorption kinetic rate constants of the model were achieved with equilibrium partition coefficient estimated by UNIFAC-ZM model. The calculated values of the model were well in consistent with experimental flux in the vacuum pervaporation of acetone, butanol and ethanol with polydimethylsiloxane membrane. The nonequilibrium model and its parameters will be further applied for prediction of separation performance and selection of operation conditions.
\end{abstract}

Keywords: Pervaporation; Nonequilibrium; Dissolution; Diffusion; Polydimethylsiloxane

\section{Introduction}

Rapid growths in population and economy have resulted in energy and water shortage on a global scale [1]. Among separation techniques for organics recovery from aqueous solutions, membrane based processes are very promising ones [2]. Membrane technology is witnessing an era of rapid growth due to the great demand of renewable energy production and water purification. Membrane pervaporation is first and mainly applied for continuous production of renewable biofuel from bio-fermentation of acetone, butanol and ethanol (ABE) aqueous solution. As an efficient technique to separate oil/water mixture, pervaporation is a permeation process through the membrane with the thermodynamic phase change. Feed liquid is passing over on one side of the membrane while the permeable component is changed to gas on the other side. Selective separation is realized via chemical potential difference between the solvents with the membrane [3]. Compared with traditional methods like distillation, adsorption, freeze crystallization, gas stripping and liquid-liquid extraction for $\mathrm{ABE}$ fermentation products recovery, pervaporation has the advantages of high selectivity, low energy consumption, moderate cost to performance ratio and compact and modular design [4]. There are several pervaporation modes, such as sweeping gas pervaporation thermos-pervaporation and vacuum pervaporation. Vacuum pervaporation is the most commonly used and investigated pervaporation configuration [5].

Many researches on polymer membrane pervaporation for $\mathrm{ABE}$ solution have been reported [6,7]. Polydimethylsilicone (PDMS) of moderate selectivity and high permeability to many organics $[8,9]$, is one of the most widely used polymer material in ABE fermentation pervaporation [10-12]. However, the separation performance of pervaporation is not high enough especially for organic permselective removal for industrial application [7]. Moreover, in spite of fact that very thin polymeric membranes are employed in several miniaturized devices, the dependence of permeability from membrane thickness is not known enough. So it is extremely important to study the mechanism of mass transfer for pervaporation, which involves various interactions between membrane and components. It is difficult to describe very precisely via present theoretical models for dense homogeneous membrane [13], including models of equilibrium dissolution diffusion [14-16], pore flow [17], virtual phase change [1820], evaporation-permeation [21] and irreversible thermodynamics [22]. Dissolution-diffusion model, the most widely accepted model, was first found by Lonsdale et al. [15], who divided pervaporation into dissolution (adsorption), diffusion and desorption three steps. From the mathematical model based on the hypothesis of equilibrium dissolution, the flux was in inverse proportion to membrane thickness and separation factor was independent of membrane thickness [23]. The conclusion was not in consistent with recent experiment results [24-27]. Pore flow model defined the dense layer as "pore" like nanofiltration which was not very reasonable, since the "pore" free volume formed by random movement of polymer chain was not fixed. Virtual phase change model was the combination of dissolution-diffusion model and pore flow model which was of some self-contradiction. Evaporationpermeation model treated the pervaporation as two separate processes, liquid evaporation and vapor permeation. The total separation factor was not equaled to the product of that two separation factors in the real operation. Irreversible thermodynamics model was set up on the chemical potential considering the coupling interaction of the

*Corresponding author: Li Jiding, State Key Laboratory of Chemical Engineering, Department of Chemical Engineering, Tsinghua University, China, Tel: +86-1062782432; E-mail: lijiding@mail.tsinghua.edu.cn

Received January 27, 2016; Accepted February 18, 2016; Published February 26, 2016

Citation: Yang X, Wu Z, Manquan F, Jiding L (2016) Nonequilibrium Dissolutiondiffusion Model for PDMS Membrane Pervaporation of ABE Water Binary System. J Membra Sci Technol 6: 143. doi:10.4172/2155-9589.1000143

Copyright: ( 2016 Yang X, et al. This is an open-access article distributed under the terms of the Creative Commons Attribution License, which permits unrestricted use, distribution, and reproduction in any medium, provided the original author and source are credited. 
components, but the phenomenological parameters needed to be determined by experiments which could not be deduced from the present theoretical models.

Pervaporation process, additional new chemical engineering operation, has many similarities to other equilibrium unit operations. However, it is not an equilibrium process in fact. Different from the main trend of equilibrium dissolution, there were few reports based on the non-equilibrium dissolution [28]. Yu et al. [29] presented nonequilibrium dissolution-diffusion model from dynamic analysis of mass transfer. The semi-experimental model, ignoring the desorption resistance, defined "apparent" mass transfer coefficient $K_{\mathrm{s}} . K_{\mathrm{s}}$ was an experimental parameter related with follow-up diffusion. Nonequilibrium model proposed by Islam [30] optimized the analysis of mass transfer in dissolution and desorption process, in which a dimensionless parameter (similar to second Damköler number) was proposed to measure for the deviation of the nonequilibrium surface reaction from equilibrium. Islam's model was further applied in recent studies of gas separation [26,31].

Above nonequilibrium hypothesis agreed with our opinion. It was considered that the dissolution equilibrium of the feed in the membrane could not be really reached in this research. Dissolution and desorption step was treated as pseudo surface reaction with the consideration of desorption resistance. The adsorption at the permeation side could be ignored with the high vacuum pervaporation method. Adsorption and desorption rate constant was applied to help for the kinetic analysis of surface reaction. The effect of thermodynamic partition and diffusion kinetic on permeation flux of acetone-water, butanol-water and ethanol-water binary system in PDMS membrane was analyzed, which could help the optimization of operation conditions, chosen of membrane material and development of theoretical model for pervaporation.

\section{Experiments}

\section{Materials}

PDMS with viscosity of $20 \mathrm{~kg} \cdot \mathrm{m}^{-1} \cdot \mathrm{s}^{-1}$ and average molecular weight of 80,000 was achieved by Beijing Second Chemistry Company of China. Acetone, butanol, ethanol, hexane and triethyl phosphate (TEP) of analytical grade were purchased from China Medicine Group (Shanghai Chemical Reagent Corporation). Crosslinking agent tetraethylorthosilicate (TEOS) of analytical grade was obtained from Beijing Beihua Fine Chemicals Company of China. Catalyst di-nbutyltin dilaurate (DBTL) was achieved from Beijing Jingyi Chemical Reagents Corporation. All the reagents were used without further purification.

\section{Preparation and characterization of PDMS membrane}

Polyvinylidene fluoride (PVDF) support layer was prepared by the dissolution of PVDF in TEP solvent to form $10 \mathrm{wt} \%$ solution, which was then casted on the non-woven fiber by spin coating method and immersed into water to induce polymer precipitation. The effect of support layer [32,33] on pervaporation flux was eliminated by reduction of the thickness of the support layer. The residual solvent was exchanged with alcohol for 5 minutes and dried at room temperature.

PDMS membrane was prepared as the way proposed by Zhan et al. [34]. Different mass of PDMS was dissolved in n-hexane and TEOS and DBTL was subsequently added. Homogeneous PDMS solution after stirring was coated on the PVDF. The thickness of PDMS layer $\delta_{\mathrm{m}}$ was controlled by the mass ratio of PDMS-solvent and characterized by SEM monitor.

\section{Evaluation of membrane pervaporation performance}

Pervaporation experiments were performed by pervaporation laboratory rig the same as reported by Han et al. [35] in our laboratory. The pressure on the back of the membrane was controlled at 100 $200 \mathrm{pa}$, which was low enough to remove the effect of vacuum degree and adsorption at the permeation side. The experiments were carried out with different membrane thickness, feed concentration of acetonewater $(\mathrm{A}-\mathrm{W})$, butanol-water $(\mathrm{B}-\mathrm{W})$, ethanol-water $(\mathrm{E}-\mathrm{W})$ and feed temperature. The composition of permeate liquid was analyzed by the gas chromatography GC-14C (Shimadzu Co. Ltd, Japan) equipped with a thermal conductivity detector.

Membrane performance of pervaporation was evaluated via permeate flux $J$ and separation factor $\alpha$. J was defined by equation (1)

$$
J=\frac{N_{m o l}}{A t}
$$

where $N_{\text {mol }}$ was permeate mole amount, $A$ membrane area and $t$ time over which the permeate sample was collected. $\alpha$ was calculated by equation (2)

$$
\alpha=\frac{c_{p, 1}}{c_{p, 2}} / \frac{c_{f, 1}}{c_{f, 2}}=\frac{J_{1}}{J_{2}} / \frac{c_{f, 1}}{c_{f, 2}}
$$

where $c_{p}$ and $c_{f}$ were concentrations in the permeate stream and in the bulk feed stream respectively, and subscript 1, 2 permselective component and the other component.

\section{Nonequilibrium dissolution-diffusion model for vacuum pervaporation}

\section{Model hypothesis, proposition and discussion}

Nonequilibrium model for pervaporation was proposed first. Concentration polarization was neglected in high flow rate of feed. So the concentration near the surface of membrane was identified the same as the main body of the liquid. Coupling effect of the components was also neglected for convenient analysis [36].

Through previous hypothesis of equilibrium dissolution [15], the mathematical model was got as equation (3),

$$
J=\frac{D_{m}}{\delta_{m}}\left(c_{f}-c_{p}\right)
$$

where $D_{\mathrm{m}}$ was diffusion coefficient in the membrane. However, for steady pervaporation process, the dissolution of liquid in membrane could not be a thermodynamic equilibrium process, or the mass transfer would not be carried on with equal chemical potential on both side of the membrane. In this research, mass transfer of dissolution and desorption was treated as pseudo surface reaction on the surface of the membrane based on the nonequilibrium hypothesis. The flux was the net amount of the reaction per time and surface of unit. Nonequilibrium model was as equation (4),

$$
J=k_{s} c_{f}-k_{d} c_{m}^{f}=\frac{D_{m}}{\delta_{m}}\left(c_{m}^{f}-c_{m}^{p}\right)=k_{d} c_{m}^{p}-k_{s} c_{p}
$$

where $c_{m}^{f}$ and $c_{m}^{p}$ were mole volume concentration contacted with feed and production in the membrane, and $k_{s}, k_{d}$ adsorption and desorption rate constants $\left(\mathrm{m} \cdot \mathrm{s}^{-1}\right)$. Rate of capture and departure from the surface depended non-linearly on the solution composition, geometrical dimension of the membrane surface and interaction between the components and membrane [37]. Partition coefficient of the components parted between the membrane and liquid, $K$, could be defined as equation (5), 


$$
K=\frac{c_{m}^{f, \mathrm{e}}}{c_{f}}=\frac{c_{m}^{p, \mathrm{e}}}{c_{p}}=\frac{k_{s}}{k_{d}}
$$

where $c_{m}^{f, \mathrm{e}}$ and $c_{m}^{p, \mathrm{e}}$ were the equilibrium dissolution and desorption concentration of component for feed solution and production respectively. For high vacuum pervaporation, the adsorption at the permeation side with high vacuum and high flux could be ignored. The modified nonequilibrium model was as equation (6).

$$
J=k_{s} c_{f}-k_{d} c_{m}^{f}=\frac{D_{m}}{\delta_{m}}\left(c_{m}^{f}-c_{m}^{p}\right)=k_{d} c_{m}^{p}
$$

$J$ and $\alpha$ could be calculated by equation (7) and equation (8) according to the definition of equation (3) and equation (4).

$$
\begin{gathered}
J=\frac{c_{f}}{\frac{\delta_{m}}{K D_{m}}+\frac{2}{k_{s}}} \\
\alpha=\frac{\frac{\delta_{m}}{K_{2} D_{m, 2}}+\frac{2}{k_{s, 2}}}{\frac{\delta_{m}}{K_{1} D_{m, 1}}+\frac{2}{k_{s, 1}}} \frac{c_{f, 1}}{c_{f, 1}}
\end{gathered}
$$

Then $J$ and $\alpha$ of the different systems and operation conditions could be predicted with known $\delta_{\mathrm{m}}, K, D$ and $k_{\mathrm{s}}$ of both components. It could be deduced that $J$ was not in verse ratio to $\delta_{\mathrm{m}}$ and $\alpha$ was related with $\delta_{\mathrm{m}}$. The relative size of $\frac{\delta_{m}}{K D_{m}}$ to $\frac{2}{k_{s}}$ characterized the nonequilibrium degree of dissolution, which was caused by the kinetic adsorption and desorption at the membrane interface. When $\frac{\delta_{m}}{K D_{m}}>\frac{2}{k_{s}}, J=\frac{K D_{m}}{\delta_{m}} c_{f}, \alpha=\frac{K_{1} D_{m, 1}}{K_{2} D_{m, 2}} \frac{c_{f, 2}}{c_{f, 1}}$, in which the equilibrium solubility selectivity and diffusion selectivity could be characterized by $\beta=\frac{K_{1}}{K_{2}}$ and $\gamma=\frac{D_{m, 1}}{D_{m, 2}}$, respectively. The conclusion was in accordance with equilibrium dissolution-diffusion model. When $\frac{\delta_{m}}{K D_{m}}<<\frac{2}{k_{s}}$, or the membrane was very thin, $J=\frac{k_{s}}{2} c_{f}, \alpha=\frac{k_{s, 1}}{k_{s, 2}} \frac{c_{f, 2}}{c_{f, 1}}$, which meant that flux was independent of membrane thickness and separation factor was only determined by the kinetic adsorption and desorption. The kinetic adsorption rate selectivity could also be defined as $\varepsilon=\frac{k_{s, 1}}{k_{s, 2}}$.

\section{Model parameter deduction}

The model parameter $K$ was calculated by group contribution method of UNIFAC-ZM model, while $D$ and $k_{\mathrm{s}}$ were achieved by regression analysis of pervaporation experiments data.

Partition coefficient: It was not precise enough to calculate the partition coefficient as the function of solubility parameters independent of solution concentration [38]. Swelling experiment was very complicated and of high requirement of accuracy. Partition equilibrium of ABE-water binary system with PDMS was calculated via the method proposed by Huang et al. [13] in this research. UNIFAC model [39] based on the conception of group contribution was introduced to calculate the activity of penetrants in the polymer. UNIFAC model consisted of a combinatorial and a residual part. Zhong et al. [40] added a universal constant in the volume fraction expression for correction of the combinatorial part with polymer solution. Through equal of the activity in the solution and membrane, equilibrium concentration in the membrane $\left(c_{m}^{f, \mathrm{e}}\right)$ was calculated by UNIFAC$\mathrm{ZM}$ model and partition coefficients under different concentration and temperature could be deduced by Matlab program of our group. Then $\mathrm{K}$ was calculated by equation (5). $K$ and defined solubility selectivity $\beta$ under different feed concentration and temperature was showed in Figures 1 and 2. Boiling point of acetone was near $329.4 \mathrm{~K}$ at standard atmospheric pressure, so the pervaporation experiment of acetonewater was not carried on at higher temperature than $323.2 \mathrm{~K}$.

Figures 1 and 2 showed first that butanol had the largest partition coefficient $K$ and solubility selectivity $\beta$, while the water had the smallest at the same condition. Figure $1 \mathrm{a}, 1 \mathrm{~b}$ and Figure $1 \mathrm{c}$ also revealed that all the partition coefficients of $\mathrm{ABE}$ and water decreased with increasing of $\mathrm{ABE}$ concentration at $313.2 \mathrm{~K}$. At the same time, $\beta$ of acetone and ethanol decreased while butanol was on the contrary. Augment of butanol content promoted much better compatibility butanol with PDMS, along with larger increment of butanol activity in water solution than acetone and ethanol. Figure 2 illustrated that the partition coefficients of $\mathrm{ABE}$ decreased with enlargement of temperature while water was on the contrary at $5 \mathrm{wt} \%$ of feed solution. All the solubility selectivity decreased with temperature increasing. The reason might be that the increasing temperature diminished the solubility difference of $\mathrm{ABE}$ and water with PDMS besides expanding the polymer chain distance of PDMS. Separation performance at different membrane thickness, feed concentration and temperature was then analyzed according the nonequilibrium model in the following.

Diffusion coefficient and adsorption rate constant: In order to apply the nonequilibrium model for mass transfer analysis of pervaporation and diffusion coefficient and adsorption rate constant

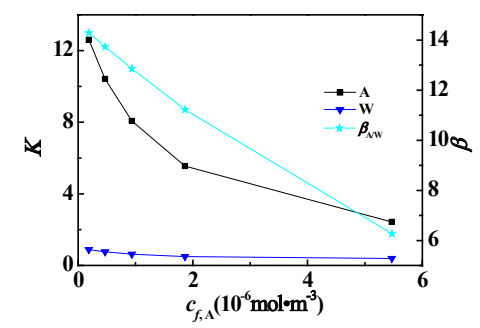

(a) A-W

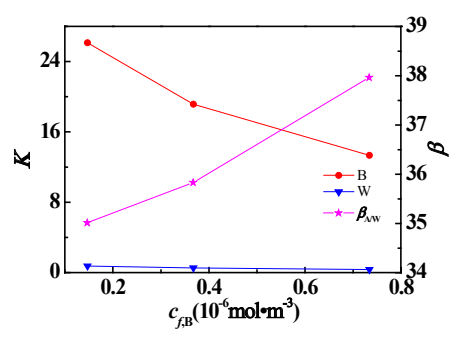

(b) B-W

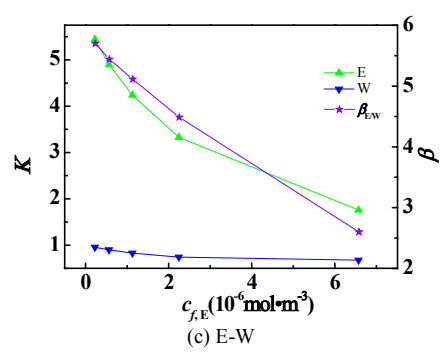

Figure 1: Partition coefficient and equilibrium solubility selectivityofdifferent concentrationof ABE solutionwith PDMS membrane pervaporation at 313.2K. 


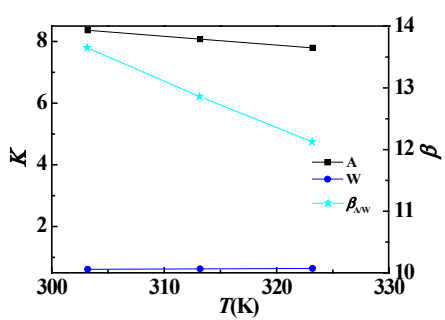

(a) A-W

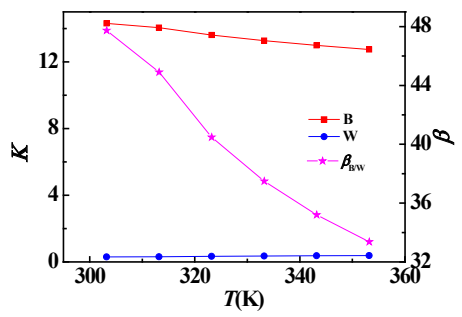

(b) B-W

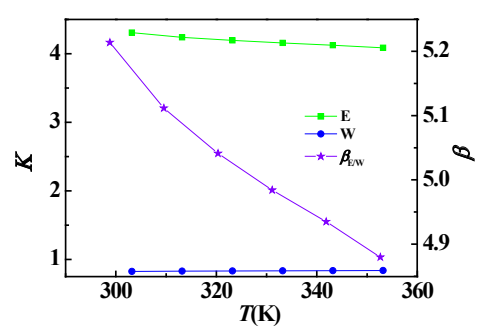

(c) $E-W$

Figure 2: Partition coefficient andequilibrium solubility selectivity of $5 \mathrm{wt} \%$ ABE solution with PDMS membrane pervaporation at different temperature.

calculation, the equation (7) was transformed to equation (9).

$$
\frac{1}{J}=\frac{\delta_{m}}{K D_{m} c_{f}}+\frac{2}{k_{s} c_{f}}
$$

Equation (9) demonstrated that $J^{-1}$ versus $\delta_{\mathrm{m}}$ gave a linear plot. According to the linear fitting of $J$ to different membrane thickness at the same concentration, $D_{\mathrm{m}}$ and $k_{\mathrm{s}}$ which were difficult to be determined directly were achieved with known $K$. Experimental data of pervaporation for $\mathrm{ABE}$ binary aqueous solution at different membrane thickness was displayed in Figure 3. The thickness of membrane was controlled in the range of 2-50 $\mu \mathrm{m}$.

Figure 3 showed that all the flux of components decreased with increasing membrane thickness, while the separation factor increased. Trade off effect between flux and separation factor occurred as in other membrane separation techniques. $J$ and $\alpha$ were both in the order, acetone $>$ butanol $>$ ethanol. When the membrane thickness was over $10 \mu \mathrm{m}, \alpha$ changed very little. That meant the dissolution process might be approximated with equilibrium process. The flux could be treated by Origin software according to Equation (9) to achieve linear fitting result as Figure 4.

Figure 4 showed $J^{-1}$ had relative good linear relationship with $\delta_{\mathrm{m}}$ as predicted by the model. When $K$ was known from Huang's method, $D_{\mathrm{m}}$ and $k_{\mathrm{s}}$ of components could be evaluated from the slope and intercept of the line, which could be used in the following prediction for the separation performance.
From Equation (9) and Figure 4, the slope of fitting line was $\frac{1}{K D_{m} c_{f}}$ and the intercept was $\frac{2}{k_{s} c_{f}}$. Then the average value of $D_{\mathrm{m}}$ and $k_{\mathrm{s}}$ of the components at $313.2 \mathrm{~K}$ determined by the nonequilibrium model with known $K$ and $c_{f}$ were listed in the Table 1 .

From Table 1, acetone had the largest $D_{\mathrm{m}}$ and $k_{\mathrm{s}}$ in the PDMS membrane, while water had the smallest $D_{\mathrm{m}}$ and $k_{\mathrm{s}}$. $D_{\mathrm{m}}$ and $k_{\mathrm{s}}$ were not only related with relative size of component and polymer molecule, but also determined by the affinity among them, which might be accused for the difference.

\section{Pervaporation performance evaluation and predicted values via nonequilibrium model}

Nonequilibrium model was then applied for the prediction of pervaporation performance with various operation conditions.

\section{Feed concentration effect}

Feed concentration was an important factor for pervaporation flux and separation factor. The concentration of $\mathrm{ABE}$ feed solution was adjusted to carry on the pervaporation experiments. Pervaporation experimental results and predicted values via nonequilibrium model with different feed concentration were shown in Figure 5. Mass concentration of butanol was controlled lower than $5 \%$ because phase separation would occur over $7 \mathrm{wt} \%$ at room temperature.

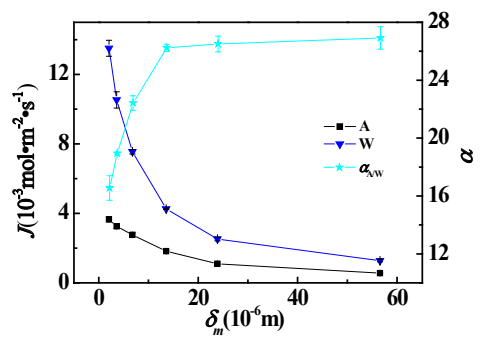

(a) A-W
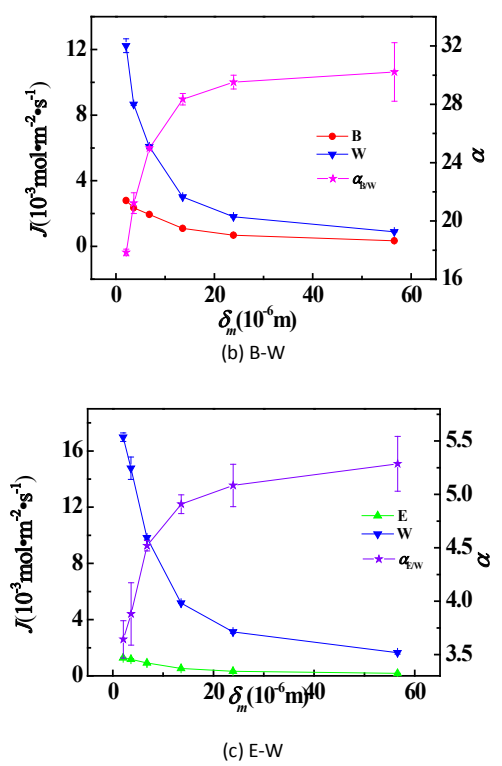

Figure 3: Flux and separation factor of $5 \mathrm{wt} \% \mathrm{ABE}$ solutionwith different Figure 3: Flux and separation factor of
thickness of PDMS membrane at $313.2 \mathrm{~K}$. 

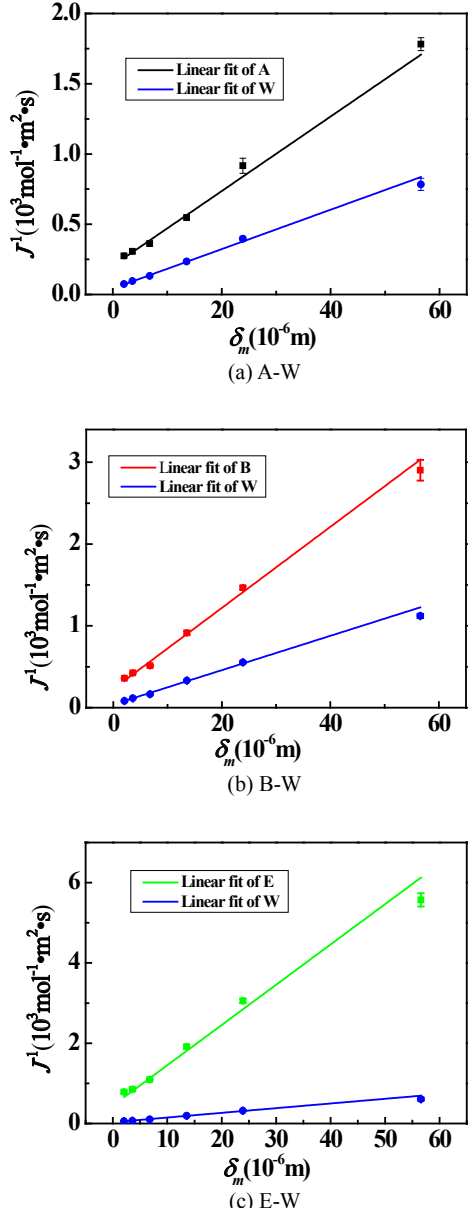

Figure 4: Linear fitting of $J^{-1}$ versus $\delta_{m}$ for $5 \mathrm{wt} \%$ ABE solution at $313.2 \mathrm{~K}$.

\begin{tabular}{|c|c|c|}
\hline Component & $\boldsymbol{D}_{\mathrm{m}}\left(\mathbf{1 0} \mathbf{0}^{-\mathbf{1 2}} \mathbf{m} \cdot \mathbf{s}^{-\mathbf{2}}\right)$ & $\boldsymbol{k}_{\mathbf{s}}\left(\mathbf{1 0}-\mathbf{6} \mathbf{m} \cdot \mathbf{s}^{-1}\right)$ \\
\hline Acetone & 6.106 & 7.722 \\
\hline Butanol & 2.589 & 6.221 \\
\hline Ethanol & 2.050 & 3.208 \\
\hline Water & 1.867 & 0.7988 \\
\hline
\end{tabular}

Table 1: Calculated $D_{\mathrm{m}}$ and $k_{\mathrm{s}}$ of acetone, butanol, ethanol and water at $313.2 \mathrm{~K}$.

From Figure 5, the pervaporation flux of $\mathrm{ABE}$ increased with increasing concentration of $\mathrm{ABE}$, while the water flux declined. Separation factors of acetone and ethanol decreased with the increasing concentration, while butanol was on the verse. It was attributed to the increasement of $K$ with high concentration illustrated in Figure $1 b$. The calculated values according to equation (7) were in moderate accordance with experimental results in low concentration $(<5 \mathrm{wt} \%)$, which explained that $k_{\mathrm{s}}$ was independent of concentration. Huge deviation of calculated and experimental results at higher concentration might be caused by the intensified swelling of organics with PDMS polymer chain.

\section{Temperature effect}

Temperature effect on the pervaporation performance was further examined. The operation temperature for pervaporation was in the range $303-353 \mathrm{~K}$ for $5 \mathrm{wt} \%$ ABE solution. The results were shown in Figure 6.
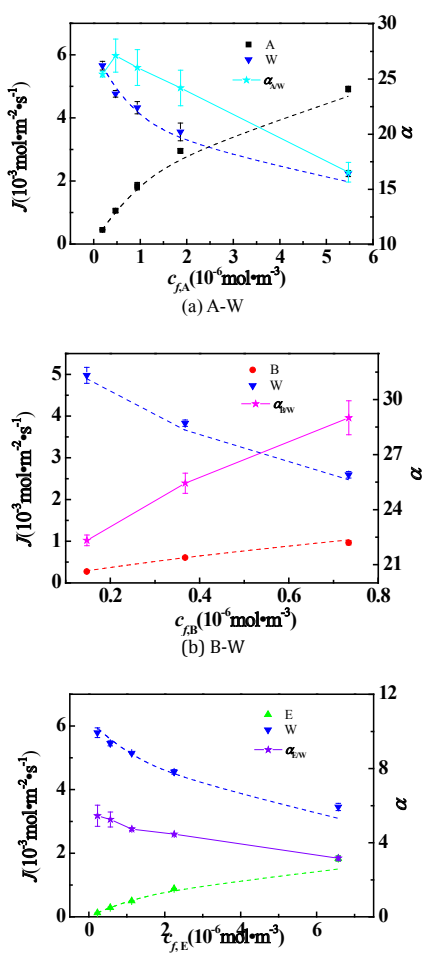

(c) E-W

Figure 5: Experimental flux, separation factor and predicted values via non-equilibrium model with different feed concentration at 313.2K (Dash line represented the model calculated results).
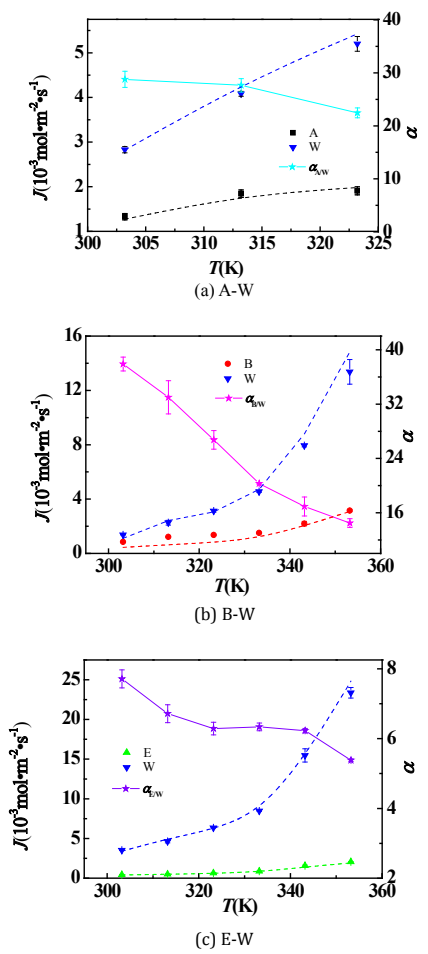

Figure 6: Experimental flux, separation factor and predicted values via non-equilibrium model for $5 \mathrm{wt} \% \mathrm{ABE}$ solution at different temperature(Dash line represented the model calculated results). 
From Figure 6, all the flux increased with temperature elevating, while the separation factor decreased. The predicted curve of nonequilibrium model agreed with the experimental results well. $D_{\mathrm{m}}$ and $k_{\mathrm{s}}$ at different temperature were determined the same as the method presented above. The change of $D_{\mathrm{m}}$ and $k_{\mathrm{s}}$ with temperature was showed in Figure 7.

As shown in Figure 7, $D_{\mathrm{m}}$ and $k_{\mathrm{s}}$ of all the components increased with higher temperature. Based on the pseudo reaction hypothesis, the kinetic adsorption process could be treated by Arrhenius fitting like diffusion as equation (11) and equation (12),

$$
\begin{aligned}
& D_{m}=D_{m, 0} e^{-\frac{E_{D}}{R T}} \\
& k_{s}=k_{s, 0} e^{-\frac{E_{s}}{R T}}
\end{aligned}
$$

where $E_{D}$ and $E_{s}$ were the diffusion and kinetic adsorption activation energy respectively, $D_{s^{\prime} 0}$ and $k_{s^{\prime} 0}$ the pre-exponential factors. Through linear fitting of $\ln D_{\mathrm{m}}$ versus $1 / T$ and $\ln k_{\mathrm{s}}$ versus $1 / T$ according to equation (11) and equation (12) in Figure 7, $E_{D}$ and $E_{s}$ were characterized by the slope of the fitting line, while the intercept characterized the $D_{s^{\prime} 0}$ and $k_{s}, 0$. The value of were collected in Table 2 .

From Table 2, water had the largest $E_{s}$ and $E_{D}$, which meant that $k_{s}$ and $D_{s}$ of water changed the most with temperature. Butanol had the smallest $k_{s^{\prime} 0}$ and $D_{s^{\prime},}$, which might be accused to the large volume of the butanol molecule. Those values would be applied for the $D_{m}$ and $k_{s}$ determination and flux calculation at the other temperature.

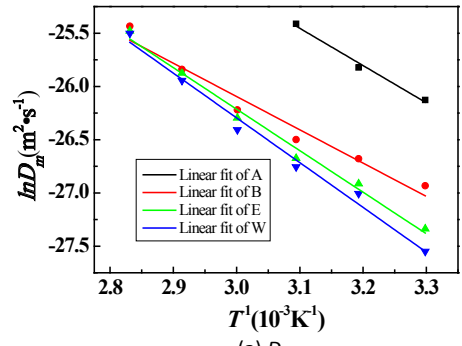

(a) $D_{\mathrm{m}}$

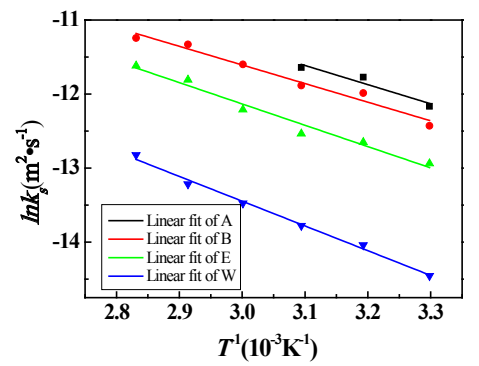

(b) $k_{\mathrm{s}}$

Figure 7: Arrhenius fitting of $D_{\mathrm{m}}$ and $k_{\mathrm{s}}$ for $5 \mathrm{wt} \% \mathrm{ABE}$ solution for $5 \mathrm{wt} \%$

\begin{tabular}{|c|c|c|c|c|}
\hline Component & $E_{s}\left(10^{3} \mathrm{~kg} \cdot \mathrm{m} \cdot \mathrm{s}^{-2}\right)$ & $E_{D}\left(10^{3} \mathrm{~kg} \cdot \mathrm{m} \cdot \mathrm{s}^{-2}\right)$ & $k_{\mathrm{s}, 0}\left(10^{-2} \mathrm{~m} \cdot \mathrm{s}^{-1}\right)$ & $D_{\mathrm{m}, 0}\left(10^{-7} \mathbf{m} \cdot \mathbf{s}^{-2}\right)$ \\
\hline Acetone & -2.588 & -3.497 & 2.753 & 4.504 \\
\hline Butanol & -2.516 & -3.128 & 1.729 & 0.5533 \\
\hline Ethanol & -2.881 & -3.901 & 3.046 & 4.994 \\
\hline Water & -3.344 & -4.204 & 3.285 & 11.41 \\
\hline
\end{tabular}
$A B E$ solution at different temperature.

Table 2: Calculated $E_{\mathrm{s}}, E_{D}, k_{\mathrm{s}, 0}$ and $D_{\mathrm{m}, 0}$ of acetone, butanol, ethanol and water.

\section{Conclusions}

In our pervaporation research, the dissolution and desorption steps were not equilibrium processes, which was different from traditional opinions and proved by the separation factor changing with membrane thickness. So the dissolution and desorption was treated as pseudo surface reaction in this work and nonequilibium dissolution-diffusion model for pervaporation membrane separation was built and analyzed. Membrane thickness, partition coefficient, diffusion and adsorption velocity were all related with flux from the semi-experimental model. Only the thickness of the membrane was very thick or the dissolution-desorption was extremely fast, the mass transfer resistance of dissolution and desorption could be ignored. After partition coefficient achieved by UNIFAC-ZM model and diffusion coefficient and adsorption rate constant regressed from the experiments, the model was applied in prediction of pervaporation separation for low organics concentration of acetone-water, butanol-water and ethanolwater with PDMS membrane. The operation conditions like membrane thickness, feed concentration and temperature were all examined. The experimental results were in good accordance with model calculation. Kinetic solubility process, as well as diffusivity, affected strongly the overall permeation and separation behavior of a PDMS membrane, which could thus be a strong function of the penetrant. Though the nonequilibrium dissolution - diffusion model is not very popular at present, this discussion might introduce the interest of research with membrane surface and dissolution kinetics in future. Those thermodynamics and kinetic parameters could not only be applied for the further performance prediction and material selection, but also offer the data to the further theoretical models for gas separation, pervaporation and reverse osmosis based on the same nonequilibrium mechanism.

\section{Acknowledgement}

We highly appreciated the financial supports of Jiangsu National Synergetic Innovation Center for Advanced Materials (SICAM), National Natural Science Foundation of China (21576150), Science Foundation of Tsinghua University (20131089399) and the Special funds for technological development research of Research Institutes from National Ministry of Science and Technology (2013EG111129)

\section{References}

1. Shannon MA, Bohn PW, Elimelech M, Georgiadis JG, Mariñas BJ (2008) Science and technology for water purification in the coming decades. Nature 452: 301-310.

2. Kujawska A, Kujawski J, Bryjak M, Kujawski W (2015) Removal of volatile organic compounds from aqueous solutions applying thermally driven membrane processes. 1. Thermopervaporation. Chem Eng Process 94: 62-71.

3. Padaki M, Surya Murali R, Abdullah MS, Misdan N, Moslehyani A, et al (2015) Membrane technology enhancement in oil-water separation. A review, Desalination 357: 197-207.

4. Chapman PD, Oliveira T, Livingston AG, Li K (2008) Membranes for the dehydration of solvents by pervaporation. J Memb Sci 318: 5-37.

5. Mortaheb HR, Ghaemmaghami F, Mokhtarani B (2012) A review on removal of sulfur components from gasoline by pervaporation. Chem Eng Res Des 90: 409-432.

6. Rozicka A, Niemistö J, Keiski RL, Kujawski W (2014) Apparent and intrinsic properties of commercial PDMS based membranes in pervaporative removal of acetone, butanol and ethanol from binary aqueous mixtures. J Memb Sci 453: 108-118.

7. Kujawski J, Rozicka A, Bryjak M, Kujawski W (2014) Pervaporative removal of acetone, butanol and ethanol from binary and multicomponent aqueous mixtures. Sep Purif Technol 132: 422-429.

8. Cocchi G, De Angelis MG, Doghieri F (2015) Solubility and diffusivity of liquids for food and pharmaceutical applications in crosslinked polydimethylsiloxane 
Citation: Yang X, Wu Z, Manquan F, Jiding L (2016) Nonequilibrium Dissolution-diffusion Model for PDMS Membrane Pervaporation of ABE Water Binary System. J Membra Sci Technol 6: 143. doi:10.4172/2155-9589.1000143

(PDMS) films: II. Experimental data on mixtures. J Memb Sci 492: 612-619.

9. Cocchi G, De Angelis MG, Doghieri F (2015) Solubility and diffusivity of liquids for food and pharmaceutical applications in crosslinked polydimethylsiloxane (PDMS) films: I. Experimental data on pure organic components and vegetable oil. J Memb Sci 492: 600-611.

10. Romdhane IH, Danner RP (1993) Polymer-Solvent Diffusion and Equilibrium Parameters By Inverse Gas-Liquid-Chromatography. AIChE J 39: 625-635.

11. Zhao CW, Li JD, Jiang Z, Chen CX (2006) Measurement of the infinite dilution diffusion coefficients of small molecule solvents in silicone rubber by inverse gas chromatography. Eur Polym J 42: 615-624.

12. Zhao S, Zhang WW, Zhang F, Li B (2008) Determination of Hansen solubility parameters for cellulose acrylate by inverse gas chromatography. Polym Bull 61: 189-196.

13. Huang J, Li J, Chen J, Zhan X, Chen C (2009) Pervaporation Separation of $\mathrm{N}-\mathrm{Heptane/Organosulfur}$ Mixtures With PDMS Membrane: Experimental and Modelling. Can J Chem Eng 87: 547-553

14. J.G.CE.B. Wijmans, Baker RW (1995) The solution-diffusion model: a review. J Memb Sci 107: 1-21.

15. Lonsdale HK, Merten U, Riley RL (1965) Transport properties of cellulose acetate osmotic membranes. J Appl Polym Sci 9: 1341-1362.

16. Valentínyi N, Cséfalvay E, Mizsey P (2013) Modelling of pervaporation: Parameter estimation and model development. Chem Eng Res Des 91: 174-183.

17. Okada T, Matsuura T (1991) A new transport model for pervaporation. J Memb Sci 59: 133-149.

18. Chang CL, Hsuan C, Chang YC (2007) Pervaporation performance analysis and prediction - using a hybrid solution-diffusion and pore-flow model. Journa of the Chinese Institute of Chemical Engineers 38: 43-51.

19. Shieh JJ, Huang R (1998) A pseudophase-change solution-diffusion model for pervaporation. II. Binary mixture permeation, Sep Sci Technol 33: 933-957.

20. Shieh J, Huang RYM (1998) A Pseudophase-Change Solution-Diffusion Mode for Pervaporation. I. Single Component Permeation, Sep Sci Technol 33: 767785.

21. Wijmans JG, Baker RW (1993) A simple predictive treatment of the permeation process in pervaporation, J Memb Sci 79: 101-113.

22. Kedem O (1989) The role of coupling in pervaporation. J Memb Sci 47: 277

23. Brun JP, Larchet C, Melet R, Bulvestre G (1985) Modelling of the pervaporation of binary mixtures through moderately swelling, non-reacting membranes. J Memb Sci 23: 257-283.

24. Chen GQ, Scholes CA, Doherty CM, Hill AJ, Qiao GG, et al. (2012) The thickness dependence of Matrimid films in water vapor permeation. Chem Eng J 209: 301-312.
25. Vicinanza N, Svenum I, Næss LN, Peters TA, Bredesen R, Borg A, et al. (2015) Thickness dependent effects of solubility and surface phenomena on the hydrogen transport properties of sputtered Pd77\%Ag23\% thin film membranes. J Memb Sci 476: 602-608.

26. Firpo G, Angeli E, Repetto L, Valbusa U (2015) Permeability thickness dependence of polydimethylsiloxane (PDMS) membranes. J Memb Sci 481: 1-8.

27. Flynn EJ, Keane D, Holmes JD, Morris MA (2012) Unusual trend of increasing selectivity and decreasing flux with decreasing thickness in pervaporation separation of ethanol/water mixtures using sodium alginate blend membranes. J Colloid Interface Sci 370: 176-182.

28. Hwang S (2011) Fundamentals of membrane transport. Korean J Chem Eng 28: 1-15.

29. Yu L, Jiang W (1994) Non Equilibrium Dissolution Diffution Model of Pevaporation 45: 153-510.

30. Islam MA, Buschatz H, Paul D (2002) Non-equilibrium surface reactions-a factor in determining steady state diffusion flux. J Memb Sci 204: 379-384

31. Islam MA, Buschatz H (2005) Assessment of thickness-dependent gas permeability of polymer membranes. Indian Journal of Chemical Technology 12: $88-92$.

32. Sukitpaneenit P, Chung T, Jiang LY (2010) Modified pore-flow model for pervaporation mass transport in PVDF hollow fiber membranes for ethanolwater separation. J Memb Sci 362: 393-406.

33. Trifunović $O$, Trägårdh G (2005) The influence of support layer on mass transport of homologous series of alcohols and esters through composite pervaporation membranes. J Memb Sci 259: 122-134.

34. Zhan X, Li JD, Huang JQ, Chen CX (2010) Enhanced Pervaporation Performance of Multi-layer PDMS/PVDF Composite Membrane for Ethanol Recovery from Aqueous Solution. Appl Biochem Biotechnol 160: 632-642.

35. Han XL, Wang L, Li JD, Zhan X, Chen JA, Yang JC (2011) Separation of Ethanol from Ethanol/Water Mixtures by Pervaporation with Silicone Rubber Membranes: Effect of Silicone Rubbers. J Appl Polym Sci 119: 3413-3421.

36. She M, Hwang S (2006) Effects of concentration, temperature, and coupling on pervaporation of dilute flavor organics. J Memb Sci 271: 16-28.

37. Wang D, Gou SY, Axelrod D (1992) Reaction rate enhancement by surface diffusion of adsorbates. Biophys Chem 43: 117-137.

38. Brookes PR, Livingston AG (1995) Aqueous-aqueous extraction of organic pollutants through tubular silicone rubber membranes. J Memb Sci 104: 119-137.

39. Gmehling J, Constantinescu D, Schmid B (2015) Group Contribution Methods for Phase Equilibrium Calculations. Annu Rev Chem Biomol Eng 6: 267-292.

40. Zhong C, Sato Y, Masuoka H, Chen X (1996) Improvement of predictive accuracy of the UNIFAC model for vapor-liquid equilibria of polymer solutions. Fluid Phase Equilibria 123: 97-106. 